Proc. Indian Acad. Sci. (Earth Planet. Sci.), Vol. 90, Number 1, March 1981, pr. 85-89. (C) Printed in India.

\title{
A simple dynamic method for the evaluation of the piezoelectric activity of rock samples
}

\author{
Y SREEDHAR MURTHY, S MURALI and \\ V L S BHIMASANKARAM \\ Centre of Exploration Geophysies, Osmania University, Hyderabad 500007 , India
}

MS roceived 28 July 1980; revised 21 February 1981

\begin{abstract}
Laboratory determination of the piezoelectric activity (PEA) of geological samples is a prerequisite for the piezoelectric method of geo?hysical prospecting in the search for mineral deposits associated with quartz. The more commonly used laboratory methods, both static and dynamic, are not convenient from several considerations.

A simple method for PEA estimation of rocks which retains the advantages of both dynamic as well as static methods while dis rensing with their drawbacks is described in this paper. It is well suited for large and representative geological samples, while the experimental conditions are comparable to those met with under field conditions.
\end{abstract}

Keywords. Piezolectric activity; transient mechanical method; quartz veins; rocks.

\section{Introduction}

The piezoelectric method of geophysical prospecting is one among a new group of exploration techniques in which unconventional physical properties of media are utilised (Volarovich and Sobolev 1969). This method is applicable in prospecting for minerals and ores such as gold, mica, wolfram, etc.; occurring in association with quartz veins located in varied host rocks. Laboratory measurement of piezoelectric activity (PEA) of geological samples thus assumes great importance in the related prospecting investigations (Murali et al 1980).

At present, some methods (both static and dynamic) of laboratory determination of piezoelectric response of geological samples are in use (Parkhomenko 1971). However, these laboratory methods operate under conditions entirely dissimilar from the field conditions prevalent in the piezoelectric method of prospecting. In the present paper a simple method for the evaluation of PEA of rock samples in the laboratory under conditions comparable to those in the field is described. This method also retains the advantages of both static as well as dynamic methods. The PEA of some samples of quartz and granite were measured using this method and the results indicate the applicability of piezoelectric prospecting in locating quartz veins. 


\section{Earlier work}

The pjezoelectric effect is a characteristic property exhibited by certain materials and is manifested as the generation of electrical polarization when a mechanical force is applied to the sample. Dielectric minerals with suitable crystal structure or rocks containing such minerals with an appropriate texture also exbibit this property (Schubnikov 1946).

The piezoelectric activity of geological media in the context of prospecting seems to have been studied only since the early fifties of the present century in USSR (Volarovich and Parkhomenko 1954), even though investigation of the PEA of chemical compounds, chiefly in the form of crystals, was conducted by physicists as early as 1880 utilising both static and dynamic methods. In the static methods of estimating PEA the sample is subjected to variable degrees of compression or tension and the charge developed across faces of the sample is measured. Here the rate of build-up of pressure on the sample as well as leakage of resultant electrical charge on the surface of the sample could cause serious variations in the coefficients measured. On the other hand in dynamic methods, a continuous elastic wave source operating on small samples, usually at sonic or ultrasonic frequencies, is generally employed. The resulting voltage across the sample as compared against a standard is used in arriving at the coefficient of piezoelectric activity. The extrapolation of these results obtained to real geological situations where large dimensions and low frequencies predominate is likely to be accompanied by considerable errors (Parkhomenko 1971).

Thus, we find that, in order to study the PEA of geological objects it is desirable (a) to employ a dynamic method suited for relatively large samples which need not be precisely cut to size or trimmed, (b) to choose a range of frequency of operation which corresponds to the operational frequencies in the field as well as the optimal frequencies of response of objects in question and (c) to design a suitable method of excitation to cover the entire range from highly active to least active specimens with minimum interference from problems of dynamic range, introduction of noise, etc. The authors have attempted to design an experimental technique that answers most of the above, if not all requirements.

\section{Experimental set-up}

The lithologically representative samples chosen for the present study have dimensions of the order of $20 \times 10 \times 10 \mathrm{~cm}$ to $40 \times 30 \times 30 \mathrm{~cm}$ or more. Rectangular blocks with more or less even surfaces were found to be adequate for the experiment. The present experimental technique (shown in figure 1) has a significant advantage over the earlier approaches mentioned in that the PE response is studied in a 'white band' of mechanical vibrations and not at any single frequency or given range of frequencies when the body is under forced oscillation. This is achieved by a transient mechanical signal generated by the impact of hammer or a dropping weight on the sample producing a single sharp pulse (Dobrin 1976). The mechanical energy imparted to the rock sample causes an elastic deformation in it. This in turn gives rise to a piezoelectric field, the electrical component of which is picked up by two copper electrodes placed on the surface of the sample. The electrical signal picked up is passed through an 


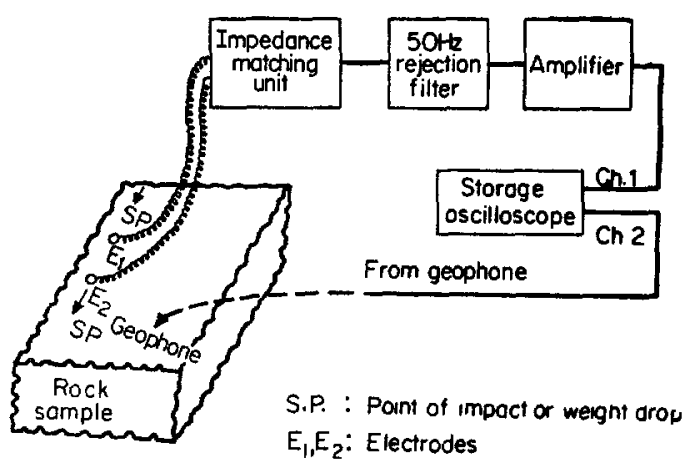

Figure 1. Experimental set-up for observing the piezoelectric activity of ruck samples.

impedance matching buffer stage, a mains rejection filter and subsequent amplification adjustable at $1: 1$ and $1: 40$. The piezoelectric response is then recorded on one channel of a storage oscilloscope. While the electrical component could thus be recorded and expressed in volts, the magnitude of the mechanical impact giving rise to the electrical signal could be measured precisely using a suitable transducer or estimated by other means. We have utilised both the methods in this experiment.

In the first case, an electromechanical transducer was placed within a short distance $(5 \mathrm{~cm})$ from the point of impact while ensuring good coupling with the sample. The transducer was an electromagnetic one with a natural frequency of $10-11 \mathrm{~Hz}$ and a coefficient of electromechanical coupling of $0.7 \mathrm{volts} / \mathrm{cm} / \mathrm{sec}$. The mechanical impact could thus be expressed in volts as referred to the transducer out-put and was recorded on a second channel of the oscilloscope. Records were obtained for different magnitudes of mechanical impact and a relation between the magnitude of mechanical impact and the resultant electrical signal voltages for a vein quartz sample could be established (figure 2a).

In the second case, generation of elastic deformation and the resultant PE signal was obtained by dropping a spherical weight at a fixed point on the sample from heights varying from $20-90 \mathrm{~cm}$ at intervals of $5-10 \mathrm{~cm}$ above its upper surface. Due care was taken to see that the weight was always dropped at the same spot on the sample. Repeat weight drops were made to facilitate the establishment of the signal reliability. The mutual relation between mechanical energy and the electrical signal voltage obtained in this way for the quartz sample are shown in figure $2 \mathrm{~b}$, assuming that the collision of the weight with the rock sample to be sufficiently elastic.

Comparing the results of hammering and weight dropping on the quartz sample (figures $2 a$ and $b$ ) it is observed (i) that the relation between the piezoelectric signal amplitude and the mechanical energy input can be represented by a linear function and (ii) that the transducer reaches saturation for the mechanical energy equal to the weight drop from $40 \mathrm{~cm}$ (which corresponds to an energy of $5.05 \times$ $10^{6} \mathrm{ergs}$ ) thus affecting the linearity of the curve for greater energies. The weight dropping method is however free from this drawback. The slope of the straight 


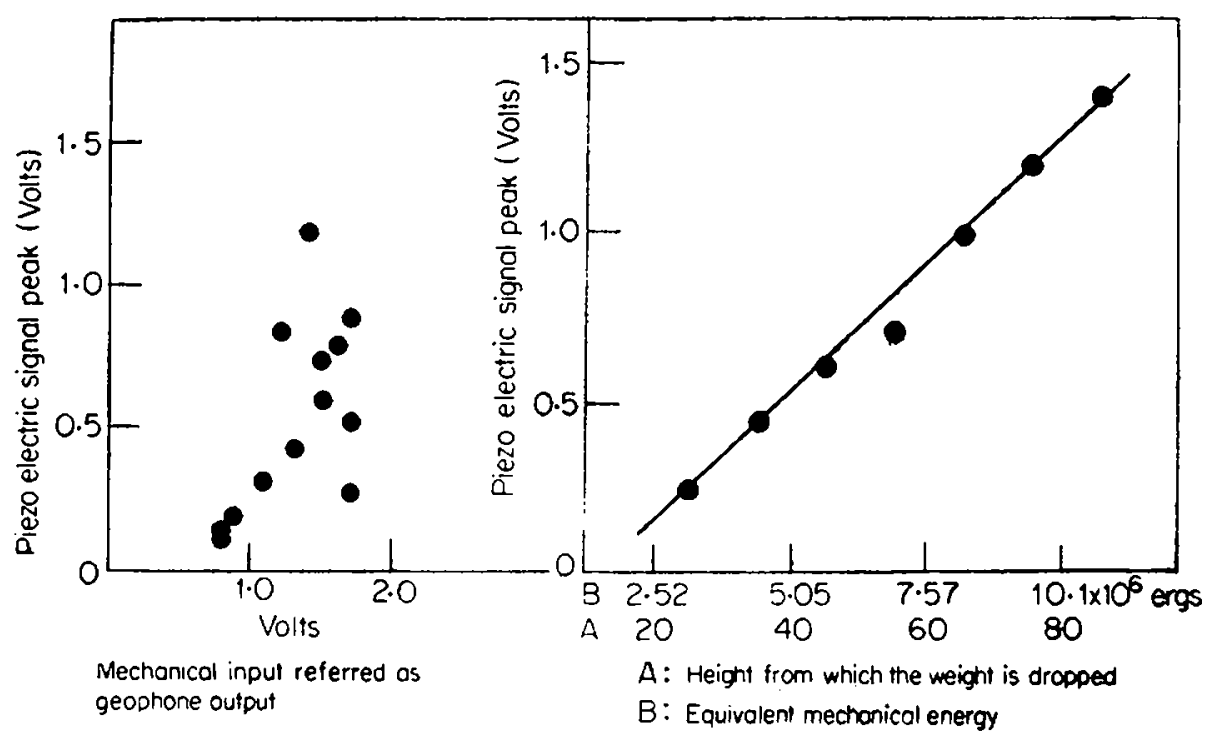

Figure 2. Piezoelectric response of vein quartz.

line is characteristic of the sample and the net PEA of samples could thus be estimated from

$$
\mathrm{C}_{\mathrm{pEA}}=\frac{\text { electrical signal amplitude in volts }}{\text { mechanical signal }} \quad 1 / l \mathrm{cgs} \text { units }
$$

where $l$ is the distance between the measuring electrodes.

A specimen record of the electrical transient is shown in figure 3. It may be seen here that the electrical signal has an oscillatory character and the peak amplitudes decay with time. Hence only the first peak amplitude is utilised in computing the coefficient of PE activity. Further it is also observed that the PE signal has a predominant frequency. The frequency of the electrical signal is independent of the amplitude of the mechanical signal or of its nature (whether weight drop or hammer source) and appears to be related to the physical dimensions of the sample only. Results of the estimation of PEA of four samples of quartz and granite obtained in this fashion are given in table 1 . These values of PEA are in close conformity with the values reported by Kondrashev (1970) for quartz and granite samples. The results indicate that PEA coefficients differ by a magnitude of a few orders between quartz and granite (which is next in order of PE activity). Based on this we propose the piezoelectric method for the direct detection of buried quartz veins in granitoid or other crystalline country rocks which is rather difficult by any of the conventional geophysical methods.

\section{Conclusions}

Weight dropping on samples produces a mechanical signal capable of generating a PE signal in a wide variety of geological samples. The proposed method is convenient for the estimation of PEA of rocks since roughly cut geological samples 


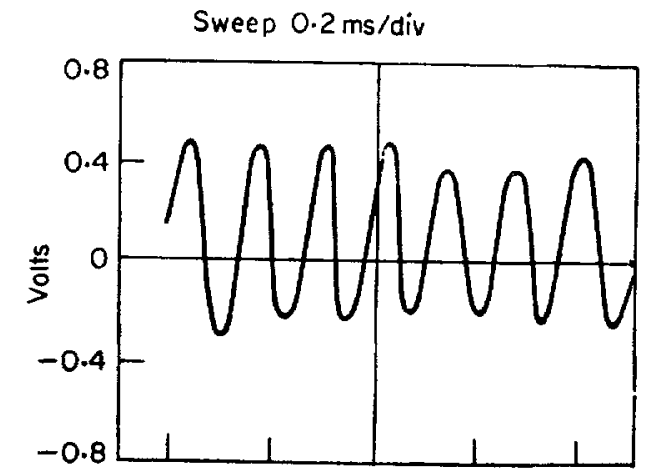

Figure 3. Blectrical transient record.

Table 1. Coefficient of piezoelectric activity of quartz and granite samples.

\begin{tabular}{llccc}
\hline No. Sample & Location & $\begin{array}{c}\text { Sample } \\
\text { volume } \\
\mathrm{cm}^{\mathrm{s}}\end{array}$ & PEA $\times 10^{8}$ & $\begin{array}{c}\text { Frequency } \\
\mathrm{kHz}\end{array}$ \\
\hline 1. Vein quartz & Choulapalli & 19,470 & $3 \cdot 29$ & $4 \cdot 1$ \\
2. Granite (grey) & Hyderabad & 10,360 & $0 \cdot 55$ & $4 \cdot 2$ \\
3. Granite (grey) & Hyderabad & 6,900 & $0 \cdot 97$ & $5 \cdot 2$ \\
4. Vein quartz & Choulapalli & 2,800 & $3 \cdot 10$ & $8 \cdot 7$ \\
\hline
\end{tabular}

of fairly large size can be used without any earlier preparation and the experiment is quite fast. While relative measurements can be made with good repeatability with this simple set-up, there is scope for absolute measurements under sufficiently controlled conditions.

\section{Acknowledgements}

The authors wish to thank the University Grants Commission for its aid in conducting the above work as part of a project.

\section{References}

Dobrin M B 1976 Introduction to geophysical prospecting (New York: McGraw-Hill) Kondrashev S N 1970 Piezoelectric method of prospecting (Moscow: Nedra) (in Russian) Murali S, Sreedhar Murthy Y and Bhimasankaram V L S 1980 Curr. Sci. 49652 Parkhomenko E I 1971 Electrification phenomena in rocks (New York: Plenum Press) Schubnikov A V 1946 Piezoelectric Textures (Akad. Nauk. SSSR) Volarovich M P and Parkhomenko E I 1954 Dokl-akad. Nauk. SSSR 90 No, 2 Volarovich M P and Sobolev C A 1969 Geoexploration 7241 